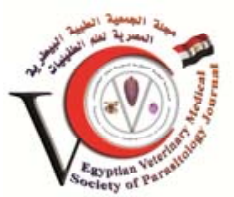

Original Article

\title{
Immunization of chicken by sonicated Eimeria acervulina
}

Lilian F Melika, Ragab M Al-Khatib

Veterinary Serum and Vaccine Research Institute, Cairo, Egypt

\begin{abstract}
:
Through this recent study sonication of the previously sporulated Eimeria acervulina oocysts were used for immunization purpose. A total number of 80 susceptible chicks were divided into four groups (A, B, C and D, 20 chicks each), the first three groups received sonicated sporulated oocysts of Eimeria acervulina in dose of 30000, 40000 and 50000 per so /one chick respectively while group $\mathrm{D}$ was left without immunization as a control group. The first three groups withstand challenge test using hot strain of Eimeria acervulina and showed protection percentage of 70, 80 and $85 \%$ respectively with minimal lesion score, obvious increase body weight gain and good health condition compared with the control group which recorded a high level of score lesion up to +4 with $0.0 \%$ protection and a high mortality rate. Also observed reduction in fecal oocysts shedding in immunized chicks compared with the control one. On applying ELISA test for confirmation it was found that there was increasing in antibody titer against Eimeria acervulina in immunized birds compared with those of the control group.
\end{abstract}

Key words: ????????????????

\section{INTRODUCTION}

Poultry coccidiosis caused by protozoan parasites of genus Eimeria has been known for over 130 years. This disease still cause great losses in poultry meat production worldwide and characterized by bloody diarrhea and high mortality especially at young age (Hiriani and Hasnani 2005). The severity of the disease depend on the both Eimeria species and size of the infecting dose of oocysts .Nine types of Eimeria are distributed in the content, one of them is Eimeria acervulina caused by protozoon of phylum Apicomplexa, genus Eimeria which make infection in the epithelial cells of the anterior portion of the small intestine mainly the duodenum of the chicken (Vegad, 2005). The classical clinical symptoms of coccidiosis including diarrhea tinged with blood, stunted growth, emaciation, depression, ruffled feathers, anorexia, off food and obvious weight loss, the disease transmitted through the food and water which got contaminated with the droppings of infected birds also rodents, cockroaches, pets, wild birds and attendants acted as a mechanical carrier of infection (Kumar, 2008). Immunization with Eimeria acervulina showed significantly decreased intestinal lesion score compared with control group, as the vaccine was given orally the antibodies produced was principally of IgA that protect the immune chicks from challenge (Sung et al., 2012).
Sonication is the act of applying sound energy to agitate particles for various purpose (Suslick, 1990) and considered a very efficient and reliable tool for cell lysis in buffer solution (Barhami, 2006). Three groups of sporulated oocysts contained 30000, 40000 and 50000 oocysts per $\mathrm{ml}$ had counted according to Jain and Archana (2011) and were used for immunization purpose.

So the aim of this work is a trail for making a sonication of the sporulated oocysts of Eimeria acervulina for the immunization of young Coccidia free chicks and determines its potency using challenge and serological tests.

\section{MATERIALS AND METHODS}

Strain used:

Eimeria acervulina strain used in this study was kindly provided from Parasitological Vaccine Research Department, Veterinary Serum and Vaccine Research Institute Cairo, Egypt.

\section{Propagation and isolation of Eimeria acervulina oocysts for experiment:}

Twenty young 7-day age chicks, 5 chicks each were inoculated orally by Eimeria acervulina sporulated oocysts (previously isolated through the Parasitological Vaccine 
Research Department, VSRI), in a dose of 5000 oocysts/ one chick. The dropping of the experimentally infected chicks were collected for six days starting from the $4^{\text {th }}$ days post infection, soaked in the water overnight and the fecal material was ground and homogenized by mortar according to Dulloul et al. (2002). The oocysts were collected from the fecal samples using flotation technique please see Figure (1). Also, from the predilection site which the duodenum of the experimentally infected chicks, and preserved in $2.5 \%$ potassium dichromate to induce sporulation of Eimeria acervulina to be infective then kept in refrigerator until used as described by Fatma et al. (2013). These sporulated oocysts were re-inoculated orally to another five susceptible chicks for the purpose of increasing the number of sporulated oocysts used for applying sonication

\section{Experimental design:}

Total of one hundred young chicks Saso strain were used in this study. Twenty chicks seven days age were used for making the propagation of Eimeria acervulina to obtain a huge numbers of oocysts for immunization purpose. The remaining eighty chicks two weeks ago were dividing into four groups $A, B, C$ and $D$, inoculated orally with 30000 , 40000 and 50000 sonicated sporulated Eimeria acervulina oocysts respectively, while group D was kept as control unimmunized group to detect the degree of protection after making challenge with 60000 sporulated Eimeria acervulina virulent strain . ELISA technique was carried out for the confirmation.

\section{Sonication of the sporulated oocysts:}

The sporulated Eimeria acervulina oocysts were divided into three groups contained 30000, 40000 and 50000 oocysts, washed with phosphate buffer saline $\mathrm{pH} 7.2$ and then undergo concentration by making centrifugation at $3000 \mathrm{rpm}$ for 20 minutes, then the sediment oocysts were sonicated using Sonics, Materials, USA. Through amplification $75 \%$ of the capacity of the apparatus three times two minutes interval for making lysis of the sporulated Eimeria acervulina, then kept in deep freeze until used for immunization purpose according to Akhtar et al. (1998, 2001), and Eskander and Germin (2003).

\section{Chicken immunization and challenge test:}

The 80 chicks of two weeks of age were divided into four groups; A, B, C and D as 20 chicks/group and then immunized by 30000, 40000 and 50000 sonicated sporulated Eimeria acervulina oocysts per so respectively, group D was left without immunization as control group.
All the four tested groups (A, B, C and D) were challenged three weeks post immunization using 60000 sporulated oocysts virulent strain/chick as recoded by Shirly et al. (1995), during that time fecal samples of all the immunized and challenged chicks were collected daily to detect the presence or absence of oocysts, also the dead tested birds were collected for post mortem examination for recoding the degree of the infection through determining the score lesions post challenge.

The score lesion were classified as follows; 0 absence of lesion, +1 white plaques like lesion containing developing oocysts were confirmed to the duodenum (Zebra striping obvious) +2 lesion much closer together (Zebra striping detected), +3 lesion numerous enough to cause coalescence with reduction in lesion size and gave the intestine the collected appearance (Zebra less obvious), +4 score lesion the mucosal wall was grayish with colonies completely coalescent striping not obvious please see Figure (2) according to Muthusamy et al. (2011). The survived tested chicks were slaughtered and severity of the score lesion was recorded to determine the efficacy of the prepared vaccine as described by Martin et al. (2007) and Amer et al. (2010).

\section{Evaluation of the experiment:}

Indirect ELISA test was applied after making titration of the Eimeria acervulina phenolized antigen Diluted as 1:10 in sodium carbonate buffer $\mathrm{pH} 9.7$ for $18 \mathrm{~h}$ at $4^{\circ} \mathrm{C}$, and washed three times with PBS containing 0.05\% Tween 20, Blocked with the blocking buffer as $100 \mu \mathrm{l} /$ well and incubated at $37^{\circ} \mathrm{C}$ for one hour, the serum samples after titration was used as dilution 1:20 using anti-chicken IgG whole molecule horseradish peroxides conjugate for

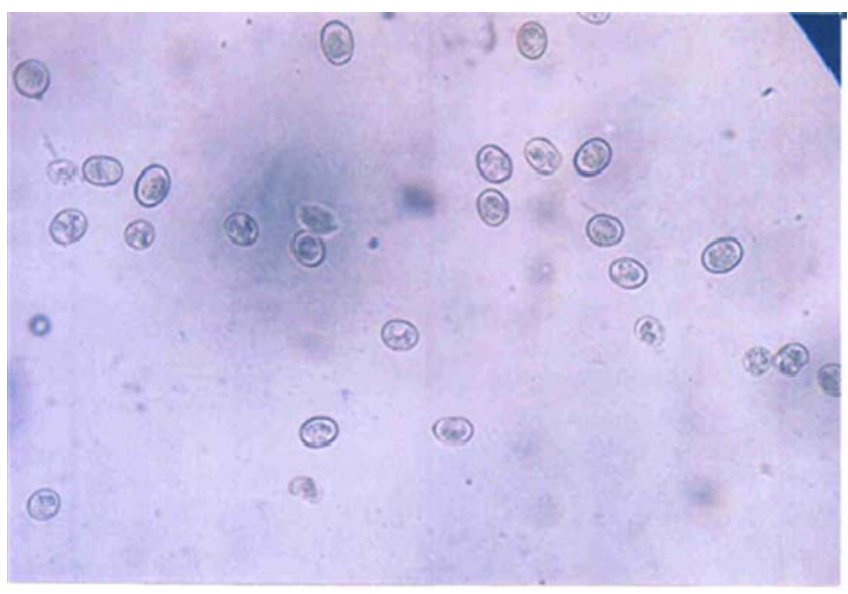

Figure 1: Purified sporulated oocysts of Eimeria acervulina isolated from the fecal samples using the flotation technique $(40 \mathrm{x})$. 


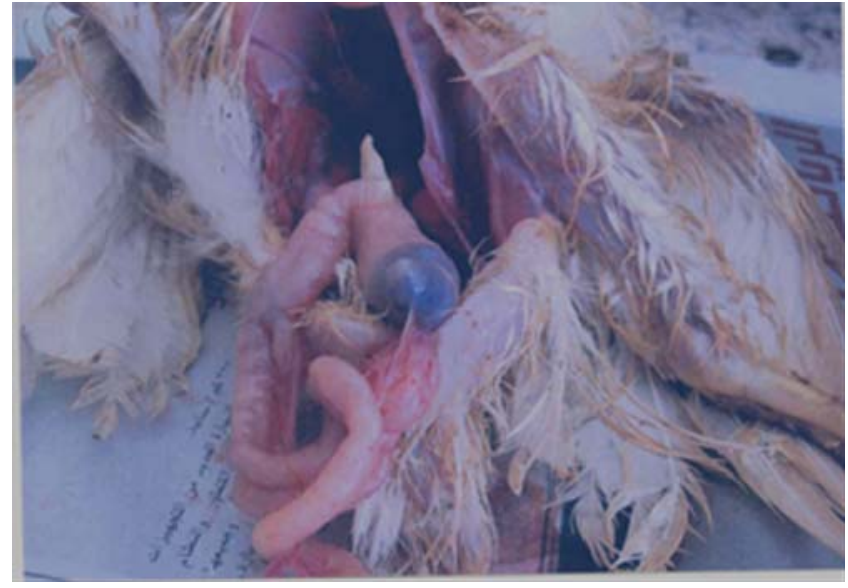

Figure 2: Score lesion +4 of the duodenum of control infected chick showed grayish mucosal wall with colonies completely coalescent and the intestinal wall is very much thickened and the intestine filled with creamy exudates containing large number of Eimeria acervulina oocysts.

measuring the antibody titer in the sera of the tested chicks post immunization and challenge test as recorded by Smith et al. (1994) and Dalloul et al (2002).

\section{Statistical analysis:}

Statistical analysis ANOVA single factor was carried out for determine the differences between the three groups of the chicks $A, B$ and $C$ in case of the oocysts output as recorded in Table (3) and the ELISA as shown in Table (5).

\section{RESULTS}

As shown in Table (1), results of the challenge test using 60000 sporulated oocysts virulent strain of Eimeria acervulina showed number of death 6,4 and 3 in case of groups A, B and C, respectively. Meanwhile, the protection rates revealed percentage 70,80 and $85 \%$ for groups $A, B$ and $C$, respectively. Control group $D$ showed no protection; all the chicks died within the first 7 days post-challenge. In addition, the degree of score lesion in groups $A, B$ and $C$ showed $+2,+1$ and 0 respectively, while group $D$ revealed highest score lesion ranged between +3 to +4 . Regarding the body weight gain there were detected normal increasing in case of the immunized three groups in comparison with the control group D.

Table (2) declares that there were no oocysts were detected in the feces of the immunized chicks through the first two weeks post immunization in case of groups A, B. and $C$ also through the first three days post challenge in the fecal samples of the all four groups (A, B, C and D), then they began to appear in case of group $A$ that immunized with 30000 sonicated sporulated oocysts , the number of oocysts were counting 21000 oocysts/gram faces on the $4^{\text {th }}$ day post immunization then decreased gradually to become $19800,15000,12000,9000,6600$, $6000,4200,2100,1200,600$ and 300 oocysts from the $5^{\text {th }}$ day to the $15^{\text {th }}$ day post-challenge, while group $B$ which immunized with 40000 oocysts/chick showed that oocysts output were measuring 13500 oocysts in the $4^{\text {th }}$ days then increased unexpectedly to 18000 oocysts in the $5^{\text {th }}$ day post challenge and then start to decrease again to become 10500, 9600, 7200, 5100, 3900, 2400, 1500, 900, 300 oocysts in the $6^{\text {th }}$ day to record $9600,7200,5100$ and 3900 on the $7^{\text {th }}$ days to the $14^{\text {th }}$ day post-challenge then disappear completely to become 0 on the $15^{\text {th }}$ day post challenge. Group C which immunized with 50000 sonicated sporulated oocysts showed 16500 oocysts in the $4^{\text {th }}$ day post challenge then the number of oocysts were decreased recording $7200,5400,4200,3000,2100$, $1800,900,300$ oocysts from the $5^{\text {th }}$ day to the $14^{\text {th }}$ day post challenge then no oocysts were detected in the $15^{\text {th }}$ day post challenge. Control group D was recording 22000 oocysts in the $4^{\text {th }}$ day post challenge to be increase to 30000,55000 and 90000 oocysts on the $5^{\text {th }}$, 6th and $7^{\text {th }}$ days respectively discharged by the control unimmunized chicks group $\mathrm{D}$ then the chicks died.

Table (4) illustrates the result of ELISA test, there were obvious increasing in the antibody titer of tested chicks of groups A, B and C through three weeks post immunization. They were recording on the third week

Table 1: Results of challenge test on young chicks immunized with sonicated sporulated Eimeria acervulina oocysts

\begin{tabular}{|c|c|c|c|c|c|c|c|}
\hline $\begin{array}{l}\text { Chicks } \\
\text { group }\end{array}$ & $\begin{array}{c}\text { No. of } \\
\text { exp. birds }\end{array}$ & $\begin{array}{c}\text { Dose of sonicated } \\
\text { sporulated oocysts/chick }\end{array}$ & Challenge dose & $\begin{array}{l}\text { No. of } \\
\text { deaths }\end{array}$ & $\begin{array}{l}\text { Score } \\
\text { lesion }\end{array}$ & $\begin{array}{l}\text { Body weight } \\
\text { gain }\end{array}$ & $\begin{array}{c}\text { Protection } \\
\%\end{array}$ \\
\hline$A$ & 20 & 30000 & & 6 & +2 & Increase & $70 \%$ \\
\hline B & 20 & 40000 & 60000 & 4 & +1 & Increase & $80 \%$ \\
\hline $\mathrm{C}$ & 20 & 50000 & $\begin{array}{c}\text { sporulated } \\
\text { Oocysts/chick }\end{array}$ & 3 & 0 & Increase & $85 \%$ \\
\hline $\mathrm{D}$ & 20 & - & & 20 & $\begin{array}{l}+3 \\
+4\end{array}$ & decrease & $0 \%$ \\
\hline
\end{tabular}


Table 2: The number of oocysts output of the chicks immunized with the sonicated sporulated oocysts in two weeks post immunization and challenge

\begin{tabular}{|c|c|c|c|c|c|c|c|c|c|c|c|c|c|c|}
\hline \multirow{2}{*}{$\begin{array}{l}\text { Chicks } \\
\text { groups }\end{array}$} & \multirow{2}{*}{$\begin{array}{c}\text { Dose of } \\
\text { sonicated } \\
\text { oocysts/chick }\end{array}$} & \multirow{2}{*}{$\begin{array}{l}\text { No of the oocysts } \\
\text { yield/gm of feces, } \\
2 \text { weeks post } \\
\text { immunization } \\
\text { and 1-3 days } \\
\text { post challenge }\end{array}$} & \multicolumn{12}{|c|}{ No of oocysts post challenge in days } \\
\hline & & & 4th & $5^{\text {th }}$ & $6^{\text {th }}$ & $7^{\text {th }}$ & $8^{\text {th }}$ & $9^{\text {th }}$ & $10^{\text {th }}$ & $11^{\text {th }}$ & $12^{\text {th }}$ & $13^{\text {th }}$ & $14^{\text {th }}$ & $15^{\text {th }}$ \\
\hline A & 30000 & 0 & 21000 & 19800 & 15000 & 12000 & 9000 & 6600 & 6000 & 4200 & 2100 & 1200 & 600 & 300 \\
\hline B & 40000 & 0 & 13500 & 18000 & 10500 & 9600 & 7200 & 5100 & 3900 & 2400 & 1500 & 900 & 300 & 0 \\
\hline C & 50000 & 0 & 16500 & 12600 & 7200 & 5400 & 4200 & 3000 & 2100 & 1800 & 1200 & 900 & 300 & 0 \\
\hline D & - & 0 & 22000 & 30000 & 55000 & 90000 & & & & Birds & died & & & \\
\hline
\end{tabular}

Table 3: Results of the statistical analysis using ANOVA test for determining the deference between the three groups A, B and $\mathrm{C}$ concerning the oocysts output post immunization and post challenge

\begin{tabular}{lllllll}
\hline Source of variation & SS & dF & MS & $F$ & $p$ value & F crit \\
\hline Between groups & 76335000 & 2 & 38167500 & 1.006051 & 0.3766 & 3.284918 \\
Within groups & $1.25 \mathrm{E}+09$ & 33 & 37937955 & & & \\
Total & $1.33 \mathrm{E}+09$ & 35 & & & & \\
\hline
\end{tabular}

Table 4: Results of ELISA mean titer of immunized and challenged chicks against Eimeria acervulina

\begin{tabular}{|c|c|c|c|c|c|c|}
\hline \multirow{2}{*}{ Chick group } & \multicolumn{3}{|c|}{ Post immunization } & \multicolumn{3}{|c|}{ Post challenge } \\
\hline & $1^{\text {st }}$ week & $2^{\text {nd }}$ week & $3^{\text {rd }}$ week & $1^{\text {st }}$ week & $2^{\text {nd }}$ week & $3^{\text {rd }}$ week \\
\hline A & 642 & 1023 & 1450 & 1345 & 1578 & 1892 \\
\hline B & 982 & 1272 & 1662 & 1662 & 2120 & 2918 \\
\hline C & 1039 & 1782 & 2255 & 1862 & 2502 & 3453 \\
\hline D & 65 & 80 & 95 & 210 & dead & dead \\
\hline
\end{tabular}

Optical density $=450$

$\mathrm{S} / \mathrm{P}$ RATIO = sample mean-NCX $\mathrm{PCX}=$ Positive control mean

PCX - NCX

$\log 10$ titer = 1, $09(\log 10 \mathrm{~s} / \mathrm{p})+3,36 \mathrm{NCX}=$ Negative control mean

Table 5: Results of the statistical analysis of the ELISA mean titer for the three groups A, B and C

\begin{tabular}{lllllll}
\hline Source of variation & SS & dF & MS & F & P-Value & F crit \\
\hline Between groups & 2057261 & 2 & 1028630 & 2.345884 & 0.129882 & 3.68232 \\
Within groups & 6577246 & 15 & 438483 & & & \\
Total & 8634506 & 17 & & & & \\
\hline
\end{tabular}

post immunization 1450, 1662 and 2255 respectively while recording 1892, 2453 and 3453 post challenge respectively, compared with the control group $D$ which recording very low titer on the first three weeks post immunization recording 65,80 and 95 to become 210 on the $1^{\text {st }}$ week post challenge then no titer were recorded after that time because the chicks were died from the virulence of the infection. On analyzing the results statistically using ANOVA test (F-test) between the three groups A, B and C, as shown in Table (3) which declared the difference between concerning the oocysts output post immunization and challenge and Table (5) recording the ELISA mean titer for the three groups and it was found that there is no significant differences $(p>0.05)$ between the three groups $A, B$ and $C$ either in oocysts out-put or ELISA test.

\section{DISCUSSION}

Making defense against Avian Coccidiosis is one of the most important objects for improving poultry industry in our country as a result of the great loss in national economy from the high rate of mortality in poultry farms 
through the infection by Coccidiosis in general. Trial for immunizing young chicks in the early age to avoid the spread of the infection in a large scale is a very good target. Eimeria acervulina is considered a moderately harmful Coccidia and cause reduction in the rate of weight gain, reducing of egg production in laying hens, meanwhile heavy infection causes high mortality McDonald and Ballingll (1983).

Although using attenuated live vaccine mainly useful for vaccination against coccidiosis but it had some disadvantages in the vaccinated birds as short duration of immunity as stated by Champman et al. (2005). Using sonication for immunization purpose is easy, and gives satisfied results on the tested chicks. There were no local lesion detected and no undesirable reactions were observed in the tested birds. Also there were no negative responses in feeding consumption and body weight gain.

The immunized young susceptible chicks showed absence of any systemic reactions so the using sonicated sporulated Eimeria acervulina for immunization is safe, this result agree with Akter et al. (2001) who used sonicated coccidial oocysts. The protection rates were 70 , 80 and $85 \%$ in groups A, B, and C respectively as recorded in Table (1) with normal increase in the body weight of the tested chicks, the number of oocysts output in the immunized chicks showed obvious decrease compared with the control group $D$, this result agrees with those of Ziomko et al. (2005) and Lilian et al. (2014) stated that the number of oocysts output were reduced in the vaccinated birds compared with unvaccinated control ones (with the vaccine of that type use in challenge) as tabulated in Table (2).

On applying ELISA for measuring the mean antibody titer against Eimeria acervulina as showed in Table (4), it was found that there was obvious increasing in the titer of the immunized chicks compared with the control one and this result parallel to Davis et al. (1985) and Eskander and Germine (2013) they prepared attenuated Eimeria tenella anticoccidial vaccine and found that there was high antibody titer of Eimeria tenella in sera of vaccinated chicks compared with the control unvaccinated group. The sonication of the sporulated Eimeria acervulina oocysts lead to production of mucosal cell membrane immune response against the infestation by Eimeria acervulina as recorded by Holmgran and Czekinsky (2005). Although there was no significant differences between the groups of chicks A, B and C statistically either in case of oocysts out-put or mean antibody titer produced but there was obvious varying in the percentage of protection between the three groups recoding 70,80 and $85 \%$ respectively.

\section{Conclusion}

Administration of sonicated sporulated Eimeria acervulina oocysts as immunogens for raising the immunity of young chicks against challenge with this type of Eimeria proved that it was efficient, safe, has no post vacinal reaction, easy in preparation and cheap. The immunized chicks showed good health condition with normal increasing in body weight gain compared with the control group. So, using the sonicated sporulated oocysts for immunization against Eimeria acervulina could be used successfully.

\section{REFERENCES}

Akhtar, M.; Azad, M.M.; Hayat, C.S. and Ashfaque, A.M. (1998): Immune responses of sonicated coccidial oocysts in chicken. Pak. J. Biol. Sci. 4:38

Akhtar, M.; Hayat, C.S.; Ashfaque, A.M.; Ayaz, M.M. and Hussain, I. (2001): Development of immunology to coccidiosis in chicken administrated sonicated coccidial vaccine. Pak. Vet. J. 21(2):61-64.

Amer, M.M.; Awaad, M.; Rabad, M.; Dablam, T.N.; Mghetas, M. and Kutkat, M.A. (2010): Isolation and identification of Eimeria from coccidiosis in chicken. J. Amer Scin. 6(10):1107-1114.

Barhami, A.M. (2006): Immune response of chicken to an experimental sonicated coccidian oocysts vaccine. Archives of Razi Institute 61(1):43-48.

Champman, H.D.; Robert, B.; Shirly, M.W. and William, R.B. (2005): Guideline for evaluating the efficacy and safety of live anticoccidial vaccines and obtaining approval for their use in chicken and Turkeys. Avian Pathol. 34 (2):279-290.

Dulloul, R.A.; Lillehoj, H.S.; Shellem, T.A. and Doerrr, H.A. (2002): Effect of vitamin A deficiency on host intestinal immune response of Eimeria acervulina in broiler chickens Poultry Science 81:1509-1515.

Davis, P.J.; Barrat, M.J.; Morgan, M.; Pafry, S.H.; MacDonold, L.R. and Joyner, L.P.(1985): Immune response of chicken to oral immunization by trickle infection with Eimeria . Research in avian coccidiosis. Proc. Georgia coccidiosis conf. 11:618-633.

Eskander, N.B. and Germin, S.S. (2013): Attenuation of Eimeria tenella by Precocious line. Zag. Vet. J. 41(3)15241530.

Fatma, M.Y; Hala A.A. and Afat A.E. (2013): Clinicopathological studies on the effect of Artemisia cina (Sheih Baladi) on coccidiosis in chicken. Porc $.6^{\text {th }}$ Conf. Vet. Res., NCR, Cairo, Egypt, p 1-14. 
Hirani, N.D. and Hasnani, J.J. (2005): Effects of season and age on the prevalence of fowl coccidiosis. Indian J. Field Veterinarians 1(2):35-38.

Holmgren, J. and Czerkinsy, C. (2005): Mucosal immunity and vaccines. Nature Medicine Supplement (11):545-553.

Jain, P.C. and Archana, J. (2011): Diagnosis. General veterinary parasitology $\mathrm{pp}$ : 182-183.

Kumar, S.D. (2008): Poultry production "Protozoal Disease" coccidiosis p:179-182.

Lilian, F.S. Melika; AL-Khatib, E.M. and Romany, M.M. (2014): Propagation and attenuation of Eimeria acervulina. EVMSPJ. 10:41-50.

MacDonald, V. and Ballingll, S. (1983): Further investigation of the pathogenicity, immunogenicity and stability of precocious Eimeria acervulina. Parasitology 86:361-369.

Martin, W.; Shirly, M.W.; Smith L. and Blake, D.P. (2007): Challenge in the successful control of the avian coccidian. Vaccine 25:5540-5547.

Muthusamy, R.; Sansudeen, S.B.; Sankaralingam, G. and Gopal, D.R. (2011): Lesion scoring technique for assessing the virulence and pathogenicity of Indian field isolates of Eimeria species. Veterinarski 81(2):259-271.
Shirly, M.W.; Bushell, Y.E.; McDonald V. and Stromberge (1995): A live attenuated vaccine for control of avian coccidiosis in broilor breeders. Vet Res. 137:453-457.

Smith N.C.; Wallach, M.; Miller, M.D.; Morgenstern, R.; Braun, R. and Eckert, J. (1994): Maternal transmission of immunity to Eimeria maxima: Enzyme Linked Immunosorbant Assay analysis of protective antibodies induced by infection. Infect. Immun. 4:1384-1357.

Sung H.L.; Hyun, S.L.; Jang, S.L.; Kyung, W.L.; Duk, K.K.; Erik, P.L.; Robert, J.Y. and Paul, J.D. (2012): Evaluation of novel adjuvant Eimeria profilin complex on intestinal host immune response against live $E$. acervulina challenge infection. Amer. Assoc. Avi. Pathog. (56):402-405.

Suslick, K.S. (1990): Sonochemistry Scince. 247:1439-1445. Usman, J.G.; Usman, N.G.; Ayi, V.K. and Hanna, T.M. : Anticoccidial resistance in poultry: A Review. New York Science Journal 4:102-109.

Vegad J.L. (2005): Protozoal Diseases, Poultry disease; A guide for farmers and poultry professionals. Chapter 17:189-199.

Ziomko, I.; Jacek, K.; Cencek, T.; Gornowicz, E.; Aleksander, S. and Ashash (2005): Prevention of broiler chicks coccidiosis using inactivated subunit vaccine coxabic. Bull. Vet. Institute bulwy 49:299-302. 